\title{
Involvement of IGF-1R-PI3K-AKT-mTOR Pathway in Increased Number of GnRH3 Neurons During Androgen-induced Sex Reversal of the Brain in Female Tilapia
}

\author{
Akari Oda \\ Toyo University \\ Sakura Inoue \\ Toyo University \\ Ryo Kaneko \\ Toyo University \\ Yasuto Narita \\ Toyo University \\ Suzuka Shiono \\ Toyo University \\ Toyoji Kaneko \\ The University of Tokyo \\ Yung-Che Tseng \\ Academia Sinica \\ Ritsuko Ohtani-Kaneko ( $\nabla$ r-kaneko@toyo.jp )
}

Toyo University

\section{Research Article}

Keywords: Androgen, female, GnRH3 neurons, IGF-1R/PI3K/AKT/mTOR pathway, sex reversal of the brain

Posted Date: June 15th, 2021

DOI: https://doi.org/10.21203/rs.3.rs-601252/v1

License: (c) (1) This work is licensed under a Creative Commons Attribution 4.0 International License.

Read Full License

Version of Record: A version of this preprint was published at Scientific Reports on February 14th, 2022.

See the published version at https://doi.org/10.1038/s41598-022-06384-4. 


\section{Abstract}

The neuroplastic mechanism of sex reversal in the fish brain remains unclear due to the difficulty in identifying the key neurons involved. Mozambique tilapia show different reproductive behaviours between sexes; males build circular breeding nests while females hold and brood fertilized eggs in their mouth. In tilapia, gonadotropin-releasing hormone 3 (GnRH3) neurons, located in the terminal nerve, regulate male reproductive behaviour. Mature males have more $\mathrm{GnRH} 3$ neurons than mature females, and these neurons have been indicated to play a key role in the androgen-induced female-to-male sex reversal of the brain. We aimed to elucidate the signalling pathway involved in the androgen-induced increase in $\mathrm{GnRH} 3$ neurons in mature female tilapia. Applying inhibitors to organotypic cultures of brain slices, we showed that the insulin-like growth factor (IGF)-1 receptor (IGF-1R)/PI3K/AKT/mTOR pathway contributed to the androgen-induced increase in GnRH3 neurons. The involvement of IGF-1 and IGF-1R in 11-ketotestosterone (11-KT)-induced development of $\mathrm{GnRH} 3$ neurons was supported by an increase in Igf-1 mRNA shortly after 11-KT treatment, the increase of GnRH3 neurons after IGF-1 treatment and the expression of IGF-1 in GnRH3 neurons. Our findings highlight the involvement of IGF-1 and its downstream signalling pathway in the sex reversal of the tilapia brain.

\section{Introduction}

Sex reversal is a well-known phenomenon in teleost fish. Some teleosts naturally change their phenotypic sex during their lifetime, while others change it in response to environmental factors or treatments with hormones.[1-3] During sex reversal, sex change occurs not only in the gonads but also in the brain, which makes it possible to elicit the opposite sex-typical reproductive behaviours. The teleost brain and their gonads are known to be highly sexually labile throughout life.[4-6] However, the molecular and neuronal mechanisms of sex reversal in the teleost brain remain unclear.

There are some difficulties in elucidating the mechanism of sex reversal in teleost brains. First, male and female fish display the characteristic reproductive behaviours of their own species. When sex reversal occurs, reproductive behaviours change according to species-specific reproductive behaviours of the opposite sex. Due to differences in the neural networks involved in species-specific reproductive behaviours before and after the reversal, different molecular and neuronal processes in the brain must occur for the respective species. Second, sex reversal is induced in response to a variety of social, physical, environmental and hormonal stimuli. Thus, the regulatory mechanism related to sex reversal in the brain also depends on the type of key stimuli that induce it. These and other differences, such as the timing of sex reversal (e.g., during development or after maturation), make it difficult to identify the molecular and neuronal mechanisms of sex reversal in different species.

In this study, we investigated the regulatory mechanism of sex reversal in Mozambique tilapia (Oreochromis mozambicus). Tilapias are important species in aquaculture. Clemens and Inslee produced all male populations of Mozambique tilapia larvae by adding 17a-methyltestosterone (MT), an artificial potent androgen, into the diet.[7] Since male tilapia grow approximately twice as fast as females, a 
monosex population of sex-reversed males is commercially produced using androgen-supplemented feed. The mechanisms underlying sex differentiation and hormone-induced sex reversal of the gonads during early development have been well studied in tilapia.[8-13] However, the mechanisms underlying sex reversal in the tilapia brain remain unsolved.

Female and male tilapia exhibit vastly different reproductive behaviours; males fight each other and build a large circular breeding nest, while females hold and brood fertilized eggs in their mouth (mouth brooding). Treatments with androgens, such as a fish natural androgen 11-ketotestosterone (11-KT), were reported to induce male-specific reproductive behaviours in most mature females within two weeks, while their gonads still contained numerous vitellogenic oocytes and the plasma concentration of $17 \beta$-estradiol (E2) remained high. [14] Thus, tilapia is a suitable model for investigating the regulatory mechanism of androgen-induced sex reversal in the brain.

Gonadotropin-releasing hormones (GnRHs) are neuropeptides responsible for reproduction in both vertebrates and non-vertebrates.[4,15-17] Advanced teleost fishes, including tilapias, have three $\mathrm{GnRH}$ subtypes (GnRH1, GnRH2, and GnRH3).[18-20] GnRH neurons in the terminal nerve (TN) of dwarf gouramis play a modulatory role in male-specific reproductive behaviour.[21, 22] Using Nile tilapia (Oreochromis niloticus), Ogawa et al. have demonstrated that $\mathrm{GnRH} 3$ is a potent neuromodulator of male sexual behaviours such as nesting and aggression.[23] They injected antiserum against GnRH3 into the third ventricle of male Nile tilapia, which resulted in significantly decreased nest-building ability, nest size, and aggressive behavior.[23] Mature male Mozambique tilapia have been shown to have more GnRH3 neurons in the TN than females.[14] Treatment with 11-KT or MT, but not E2, increased the number of $\mathrm{GnRH} 3$ neurons in mature females to a level similar to that in males, and induced male-specific reproductive behaviors.[14] Furthermore, 11-KT has also been shown to promote neurogenesis in female tilapia and generate newly proliferated GnRH3 neurons. [24] The above-mentioned findings indicate the androgen-dependent regulation of $\mathrm{GnRH} 3$ neurons and their important role in male-specific reproductive behaviours in tilapia. However, the mechanism by which androgen affects $\mathrm{GnRH} 3$ neurons is yet to be understood.

We focused on GnRH3 neurons in Mozambique tilapia with respect to female-to-male sex reversal of the brain. The promoter of the GnRH3 gene contains binding sites for many receptors, including the glucocorticoid receptor (GR) and thyroid hormone receptor (TR), but not for the androgen receptor (AR). [25] This means that androgen binding to AR does not directly affect GnRH3. The effect of androgen on $\mathrm{GnRH}$ neurons is likely mediated through other molecules, such as hormones or neurotransmitters. Considering the critical regulatory role of insulin-like growth factor 1 (IGF-1) in growth and reproduction $[26,27]$, we examined its involvement and that of its downstream pathway in the androgen-induced effects on GnRH3 neurons. This study is the first to propose a molecular mechanism by which $\mathrm{GnRH} 3$ neurons involved in male reproductive behaviours are positively regulated through the IGF-1 and IGF-1R / phosphoinositide 3-kinase (PI3K)/AKT/ mechanistic target of rapamycin (mTOR) signalling pathway during androgen-induced female-to-male sex reversal in the tilapia brain. 
To examine the effects of androgen in the tilapia brain without any influence from other organs such as the gonads and liver, we used an organotypic culture of brain slices at the TN level, and pharmacologically investigated the possible involvement of IGF-1 signalling pathways.

\section{Results}

\section{Effects of sex steroids on the number of GnRH3 neurons in cultured brain slices}

We first examined the effect of 11-KT at different concentrations and that of $10 \mathrm{nM}$ E2 on the number of $\mathrm{GnRH} 3$ neurons in tilapia brain slices. Cross-sectional slices of the brain were cut at the TN level, and the slices were further cut into left and right halves (Fig. 1). One half of each brain slice was treated with either 11-KT or E2 (11-KT- or E2-treated group) for three days, while the other half was treated with the solvent alone (control group). GnRH3 neurons were immunostained with anti-GnRH3 (Fig. 2a), and the total number of GnRH3 neurons per animal was counted from all the half slices (2-4 slices) of the brain at the TN level originating from the respective animal (Fig. 2b). Then, the mean number of GnRH3 neurons was compared between the control and 11-KT-treated groups. Figure $2 \mathrm{~b}$ shows that 11-KT treatments with $1 \mathrm{nM}$ and $10 \mathrm{nM}$ increased immunostained GnRH3 neurons, and there was a statistically significant difference between the $10 \mathrm{nM}$ 11-KT-treated and control groups (control vs. 11-KT (10 nM), $p<$ 0.05 , paired Student's $t$-test). No increase was observed in the number of GnRH3 neurons in the $10 \mathrm{nM}$ E2treated group, compared to that in the controls.

\section{Effects of IGF-1 on the number of GnRH3 neurons in cultured brain slices}

To examine the influence of IGF-1 on the number of GnRH3 neurons, the brain slices were treated with IGF-1 for three days. The number of GnRH3 neurons was compared between the control and IGF-1-treated slices originating from the same animal. Figure $3 a$ and $3 b$ show immunostained-GnRH3 neurons and the mean numbers of GnRH3 neurons per animal, respectively, in control and IGF-1-treated slices. Treatment with IGF-1 at $10 \mathrm{nM}$ showed a tendency to increase the number of GnRH3 neurons compared to that of the controls. When $100 \mathrm{nM}$ IGF-1 was added to the medium, the number of GnRH3 neurons was significantly greater than that in controls (contol vs. $100 \mathrm{nM}$ IGF-1, $p<0.05$, paired Student's $t$-test). These results revealed a stimulatory effect of IGF-1 on the number of GnRH3 neurons.

\section{Effects of 11-KT on IGF-1 mRNA expression}

Igf-1 gene expression was examined in control and 11-KT groups using cultured halves of female brain slices originating from the same animal. The expression levels of $\lg f-1 \mathrm{mRNA}$ were measured $1 \mathrm{~h}$ after treatment with $10 \mathrm{nM} 11-\mathrm{KT}$ by real-time quantitative reverse transcription qRT-PCR, and compared between 11-KT-treated and control groups (Fig. 4). The expression of Igf-1 mRNA was significantly higher in 11-KT-treated slices than in control slices (control vs. 11-KT, $p<0.05$, paired Student's $t$-test), indicating that $11-K T$ increased the expression of $\lg f-1$ mRNA in the brain. 


\section{Effects of IGF-1R inhibitor on 11-KT-induced increase in GnRH3 neurons}

Micrographs in Fig. 5a show immunostained-GnRH3 neurons (red) in four brain slice groups: control, IGF1R inhibitor (BMS-754807, $20 \mu \mathrm{M}$ )-treated, 11-KT-treated, 11-KT- and IGF-1R inhibitor-treated groups. Control and inhibitor-treated animals originated from the same animal, as shown in Fig. 1. Similarly, 11KT-treated and 11-KT- and IGF-1R inhibitor-treated groups originated from the same animal. The graphs in Fig. $5 b$ indicate the average number of GnRH3 neurons per animal in each of the four groups.

As shown in Fig. 5b, 11-KT (10 nM) significantly increased GnRH3 neurons (control vs. 11-KT; a vs. b, $p<$ 0.05 , one-way analysis of variance [ANOVA] followed by Tukey-Kramer post-hoc test). While the potent IGF-1R inhibitor alone did not affect the number of GnRH3 neurons, the combined treatment of 11-KT with an IGF-1R inhibitor suppressed the 11-KT-induced increase in the number of GnRH3 neurons (11-KT vs. 11-KT \& BMS-754807, $p<0.05$, paired Student's $t$-test). These results indicate an important role of IGF$1 \mathrm{R}$ in the $11-\mathrm{KT}$-induced increase in GnRH3 neurons.

\section{IGF-1R-immunoreactivities in GnRH3 neurons}

To investigate whether GnRH3 neurons in 11-KT-treated females express IGF-1R, a pair of adjacent frozen sections with mirror images were subjected to immunocytochemical staining with rabbit polyclonal antibodies against GnRH3 and IGF-1R. GnRH-neurons (red in Fig. 6a) showed IGR-1R immunoreactivity (green in Fig. 6b), although IGF-1R immunoreactivity was also observed in the cells without GnRH3immunoreactivities near the ventricle.

\section{Effects of PI3K/AKT/mTOR pathway inhibitors on 11-KT- induced increase in the number of GnRH3 neurons}

To examine the signalling pathway involved in the androgen-induced increase of GnRH3 neurons, one of the inhibitors examined was added to the medium simultaneously with $10 \mathrm{nM} 11-\mathrm{KT}$, and their suppressive effect on the 11-KT-induced increase of GnRH3 neurons was examined by counting immunostained GnRH3 neurons in the brain slices. The following inhibitors were examined in this study: PI3K inhibitor (LY294002), AKT inhibitor (GDC-0068), and mTOR inhibitor (rapamycin). Micrographs in Fig. 7a-c show immunostained-GnRH3 neurons (red) in four brain slice groups: control, inhibitor-treated, 11-KT-treated, and 11-KT- and inhibitor-treated groups. Control and inhibitor-treated brain slices originated from the same animals (Fig. 1). Similarly, 11-KT-treated and 11-KT- and inhibitor-treated brain slices originated from the same animal (Fig. 1). Graphs in Fig. $7 d-f$ indicate the average numbers of GnRH3 neurons per animal in the four groups.

The effect of PI3K inhibitor, LY294002) was examined (Fig. 7a and d). The increasing effect of 11-KT on the number of GnRH3 neurons was confirmed (Fig. 7d) (control vs. 11-KT, $p<0.05$, unpaired Student's $t$ test). Treatment with LY294002 at $20 \mu \mathrm{M}$ alone did not affect the number of GnRH3 neurons compared to that in the control slices. The combined treatment of 11-KT with LY294002 significantly suppressed the 
11-KT-induced increase in GnRH3 neurons (11-KT vs. 11-KT \& LY294002, $p<0.01$, paired Student's $t$-test) (Fig. 7d).

Treatment with the AKT inhibitor (GDC-0068, pan-AKT inhibitor targeting Akt1, Akt2, and Akt3) was first examined at different concentrations $(1,10$, and $20 \mu \mathrm{M})$. GDC-0068 at 10 and $20 \mu \mathrm{M}$ significantly reduced the 11-KT-induced increase in the number of GnRH3 neurons (data not shown). The effects of GDC-0068 at $20 \mu \mathrm{M}$ were then examined in four brain slice groups (Fig. 7b and e). The stimulatory effect of 11-KT on $\mathrm{GnRH} 3$ neurons was confirmed (control vs. 11-KT, $p<0.05$, unpaired Student's $t$-test). While GDC-0068 alone did not affect the number of GnRH3 neurons, the combined treatment of 11-KT with GDC-0068 suppressed the 11-KT-induced increase in the number of GnRH3 neurons (11-KT vs. 11-KT \& GDC-0068, $p$ $<0.01$, paired Student's t-test) (Fig. 7e).

The effect of the mTOR inhibitor, rapamycin $(10 \mu \mathrm{M})$, was examined (Fig. $7 \mathrm{c}$ and f). The effect of 11-KT on the number of GnRH3 neurons was confirmed (Fig. 7f) (control vs. 11-KT, a vs. b, $p<0.05$, one-way ANOVA followed by Tukey-Kramer post-hoc test). Treatment with rapamycin did not affect the number of GnRH3 neurons, but the combined treatment of rapamycin with 11-KT suppressed the 11-KT-induced increase in the number of GnRH3 neurons (11-KT vs. 11-KT- and rapamycin-treated slices, $p<0.05$, paired Student's t-test) (Fig. 7f).

\section{Effects of ERK and JAK inhibitors on 11-KT-induced increase in $\mathrm{GnRH} 3$ neurons}

The effects of the extracellular signal-regulated kinase (ERK) inhibitor (U0126) and Janus kinase (JAK) inhibitor (InSolution ${ }^{\text {TM }}$ JAK inhibitor 1) were similarly examined (Fig. 8).

The effect of 11-KT on the number of GnRH3 neurons was confirmed in both experiments (control vs. 11$\mathrm{KT}, p<0.05$, unpaired Student's t-test in Fig. 8c and one-way ANOVA followed by Tukey-Kramer post-hoc test in Fig. 8d, respectively). Treatment with U0126 $(20 \mu \mathrm{M})$ alone did not induce any significant change in the number of GnRH3 neurons. The combined treatment of 11-KT with U0126 caused a slight decrease in the number of $\mathrm{GnRH} 3$ neurons compared to that in the 11-KT-treated group. However, there was no significant difference between them (Fig. 8c).

Similarly, treatment with InSolution ${ }^{\mathrm{TM}}$ JAK inhibitor I (100 nM) alone did not affect the number of GnRH3 neurons. The combined treatment of 11-KT with the JAK inhibitor still significantly increased the number of GnRH3 neurons, compared to that in controls, as well as in JAK inhibitor-treated slices (control vs. 11KT- and JAK inhibitor-treated, JAK inhibitor-treated slices vs 11-KT- \& JAK inhibitor-treated slices, $p<0.05$, unpaired Student's $t$-test) (Fig. $8 \mathrm{~b}$ and d). Treatment with 11-KT combined with the JAK inhibitor at different concentrations $(1,10,100 \mathrm{nM}$, and $1 \mu \mathrm{M})$ were also examined. However, simultaneous treatment with 11-KT and the JAK inhibitor at all the concentrations examined did not reduce the number of GnRH3 neurons, compared to that in 11-KT-treated slices (data not shown). 
Taken together, we showed that the inhibitors of IGF-1R, PI3K, AKT, and mTOR were highly effective in suppressing the 11-KT-induced increase of $\mathrm{GnRH} 3$ neurons in brain slices originating from mature females. Contrastingly, inhibitors of ERK and JAK did not significantly suppress the effect of 11-KT on the number of GnRH3 neurons.

\section{Discussion}

\section{Role of IGF-1 and IGF-1R/PI3K/AKT/mTOR signalling pathway with respect to the effects of 11-KT on GnRH3 neurons in the female tilapia brain}

According to the present results obtained with the TN-containing brain slices of mature females (Fig. 2), the in vitro treatment with $11-\mathrm{KT}$ increased $\mathrm{GnRH} 3$ neurons, indicating that $11-\mathrm{KT}$ can increase the number of GnRH3 neurons without mediation via other organs, such as the gonad and liver. Since there are no AR binding sites in the promoter regions of GnRH3 gene in tilapia,[25] we considered that IGF-1 might mediate the action of $11-\mathrm{KT}$ on GnRH3 neurons. Our hypothesis is strongly supported by the following four findings: 1) Treatment with IGF-1 increased the number of GnRH3 neurons (Fig. 3). 2) The expression of IGF-1 mRNA increased shortly after 11-KT treatment (Fig. 4). 3) The potent IGF-1R inhibitor, BMS-754807, strongly suppressed the 11-KT-induced increase of GnRH3 neurons (Fig. 5). 4) Immunohistochemical staining with anti-GnRH3 and anti-IGF-1R antibodies revealed the co-localization of IGF-1R and GnRH3 (Fig. 6). These results support the possibility that the effect of 11-KT on GnRH3 neurons is mediated by IGF-1 produced in the brain and IGF-1R on GnRH3 neurons. Furthermore, studies using inhibitors (Fig. 5, 7, and 8) clearly indicate the contribution of the IGF-1R downstream signalling pathway, PI3K/AKT/mTOR, to the effect of 11-KT on GnRH3 neurons. Therefore, our findings indicate that the effect of $11-\mathrm{KT}$ on the number of GnRH3 neurons is mediated by the IGF-1 and IGF-

1R/PI3K/AKT/mTOR signalling pathways.

Although there were no binding sites for ARs in GnRH3 promoters,[25] the expression of AR mRNA was reported near, but not in, the TN of female medaka [28]. Ogawa and Parhar showed by single-cell gene profiling that, although Arb mRNA as well as $\mathrm{Tr}$, Gr, and $\mathrm{Er}$ mRNAs were expressed in $\mathrm{GnRH} 3$ neurons, the expression level of Arb mRNA was so low that nested-PCR was necessary to observe it.[29] Combining these results with ours, it can be proposed that the effect of 11-KT on GnRH3 neurons is mediated by other molecules such as IGF-1 and its receptor, although it cannot be completely ruled out that 11-KT affects $\mathrm{GnRH} 3$ neurons directly.

\section{The role of IGF-1 and IGF-1R/PI3K/AKT/mTOR signalling pathway in the regulatory process of $\mathrm{GnRH}$ neurons reported in other animals}


IGF-1 is known to possess powerful neuroprotective effects in promoting neuronal survival, neuronal differentiation, neurite elongation, neurogenesis, and neurite regeneration in vivo and in vitro.[30] It is a regulator of reproductive neuroendocrine function that affects the development and functions of $\mathrm{GnRH}$ neurons in the vertebrate brain, although this has been reported mainly in mammals. [31,32] GnRH perikarya express IGF-1R before puberty in mice, suggesting a potential direct anatomical locus where IGF-1 can control reproductive development and function.[31,33] Furthermore, the action of IGF-1 on IGF$1 \mathrm{R}$ in $\mathrm{GnRH}$ neurons regulates the onset of puberty in rodents.[34,35]

In fish like Mozambique tilapia, while IGF-1 and IGF-2 are mainly produced in the liver, their transcripts are expressed in various tissues including the brain.[36] The absolute amounts of Igf- 1 and Igf-2 mRNAs were also determined in many regions, such as the liver and brain of Nile tilapia.[37] In yellow catfish, the expression of Igf-1 mRNA in the hypothalamus was higher in adult males than in adult females.[38] Additionally, the effect of androgen (MT) treatment affected its expression in a sex-dependent manner; MT treatment in females increased the expression of Igf-1 mRNA.

The activation of IGF-1R leads to the stimulation of the following two major downstream signalling pathways: (i) the PI3K-AKT-mTOR pathway and (ii) Ras-Raf-MAP pathway.[30] Regarding the relationship between $\mathrm{GnRH}$ and the intracellular signalling pathway following IGF-1R, Shao et al. investigated the expression of genes and proteins related to the IGF-1 pathway in the rat hypothalamus after combined treatments with di-(2-ethylhexyl) phthalate (DEHP), a common environmental endocrine disruptor.[37] Their study indicated that DEHP might activate the hypothalamic GnRH neurons prematurely through the IGF-1 and IGF-1R/PI3K/AKT/mTOR signalling pathway to promote GnRH release. It was recently reported that administering IGF-1 significantly increased the firing rate and frequency of spontaneous postsynaptic currents in rodents, and that IGF-1R and PI3K are involved in this process.[38] These findings indicate that IGF-1 affects the activity of GnRH neurons through IGF-1R and the $\mathrm{PI} 3 \mathrm{~K} / \mathrm{AKT} / \mathrm{mTOR}$ pathways in rodents.

In teleosts, there are only a few studies on IGF-1R downstream signalling pathways in GnRH neurons. Onuma et al. revealed that IGF-1 affects the development of GnRH2 and GnRH3 through IGF-1R in zebrafish embryos.[39] They also examined whether PI3K inhibitors and/or ERK inhibitors disturbed the distribution of GnRH3 neurons in embryos. Treatment of embryos with a PI3K inhibitor (LY294002 or wortmannin) caused abnormal GnRH3 distribution similar to that in IGF signalling-deficient embryos. Contrastingly, U0126 had no effects. These results indicate that the influence of IGF-1 on the development and migration of $\mathrm{GnRH} 2$ and GnRH3 neurons was mediated through IGF-1R and the subsequent $\mathrm{PI} 3 \mathrm{~K}$ signalling pathway. According to findings regarding $\mathrm{GnRH}$ neurons in mammals and teleosts, it is highly likely that IGF-1 and the IGF-1R/PI3K/AKT/mTOR pathway play an important regulatory role in $\mathrm{GnRH} 3$ neurons in Mozambique tilapia.

In our previous studies, when 11-KT induced male-specific reproductive behaviours in mature female tilapia, GnRH3 neurons were increased in the TN region.[14,24] Moreover, newly proliferated GnRH3 neurons have been found in the TN of 11-KT-treated mature females. [24] Considering these findings with 


\section{Reduced involvement of ERK and JAK in the 11-KT-induced increase of GnRH3 neurons}

IGF-1R signalling can also activate the Ras/Raf/Mek/ERK pathway, which is broadly involved in the regulation of neuron proliferation, differentiation, and apoptosis.[40-42] The co-activation of the ERK and AKT signalling pathways has been reported to promote the survival of $\mathrm{GnRH}$ neurons or $\mathrm{Gn} 10 \mathrm{GnRH}$ cells. $[43,44]$ Additionally, positive regulation of Gnrh mRNA levels induced by kisspeptin was mediated through the activation of ERK and AKT signalling pathways in mammalian GT1-7 (a clonal cell line of mature, differentiated GnRH neurons) and GN11 (a clonal cell line with GnRH expression) cells.[45] JAK2 is necessary for the neuroendocrine control of female reproduction and development of GnRH neurons. $[46,47]$ In vitro, $\mathrm{GnRH}$ neurons and $\mathrm{GnRH}$ cell lines respond to a variety of ligands, including cytokines, which activate the JAK/signal transducers and activators of transcription intracellular signalling pathway.[48,49] However, in this study, the ERK and JAK inhibitors did not significantly suppress the 11KT-induced increase of GnRH3 neurons, indicating a reduced contribution of ERK and JAK in mediating the effect of 11-KT on GnRH3 neurons compared to the PI3K/AKT/mTOR pathway.

We focused on GnRH3 neurons, which have been reported to control male-specific reproductive behaviour in tilapia,[23] and found a possible role of IGF-1 in mediating the effect of androgen on GnRH3 neurons. IGF-1 is a very important molecule commonly used in controlling reproduction by regulating $\mathrm{GnRH}$ neurons in mammals, as well as in controlling somatic development in vertebrates. Therefore, our findings propose an attractive idea that IGF-1 and its downstream signalling pathway play an important role during the sex reversal of the brain in tilapia.

\section{Materials \& Methods}

\section{Animals}

All experimental protocols used in this study on fish were approved by the Institutional Animal Care and Use Committee of Toyo University Itakura Campus and all experiments were performed in accordance with relevant guidelines and regulations. Furthermore, the present study were performed in accordance with ARRIVE guidelines (Animal Research: Reporting in Vivo Experiments). Female Mozambique tilapia (total length, 7-10 cm; body weight, $12-20 \mathrm{~g}$ ) were maintained in fresh water at $25^{\circ} \mathrm{C}$ and used for the experiments.

\section{Brain slice cultures}

\section{Preparation of brain slices}


Brain slices at the level of the TN were prepared according to the protocol provided by Dr. Satoshi Ogawa in Prof. Ishar S. Parhar's laboratory (Monash University, Malaysia). Mature female tilapia fish were deeply anaesthetized with $0.2 \%$ 3-aminobenzoic acid ethyl ester (MS222, Sigma-Aldrich, St. Louis, MO, USA) and decapitated. The brain was removed and stored in cooled Ringer's saline containing $124 \mathrm{mM} \mathrm{NaCl}, 3 \mathrm{mM}$ $\mathrm{KCl}, 2 \mathrm{mM} \mathrm{CaCl}_{2}, 2 \mathrm{mM} \mathrm{MgSO}_{4}, 1.25 \mathrm{mM} \mathrm{NaH}_{2} \mathrm{PO}_{4}, 26 \mathrm{mM} \mathrm{NaHCO}_{3}$, and $10 \mathrm{mM}$ glucose (pH 7.35). The brain area from the olfactory bulb to the middle of the telencephalon was dissected and embedded in $4 \%$ low melting point agarose (Sigma-Aldrich) within embedding moulds. The embedding moulds were then placed on ice. After the agarose hardened ( $~ 5 \mathrm{~min})$, the embedded brain and agarose were glued to the stage of a vibratome (Dosaka-EM Co., Ltd, Kyoto, Japan) and cut into $200-\mu \mathrm{m}$ cross-sectional slices. The slices that covered all the $\mathrm{GnRH}$ neurons in the TN were collected in a dish containing Wash Medium [DMEM phenol free (Thermo Fisher Scientific, Waltham, MA, USA)] supplemented with $134 \mathrm{U} / \mathrm{ml}$ penicillin (Cosmo Bio Co., Ltd, Tokyo, Japan), 0.25 glutamate (Sigma-Aldrich), 1 × B-27 serum-free (Thermo Fisher Scientific), $0.13 \mathrm{mM}$ streptomycin (MeijiSeika Pharma, Tokyo, Japan), and 0.5 L-glutamine (SigmaAldrich)]. Each slice was then divided into equal halves by cutting at the midline (Fig. 1), and the halves were separately transferred to wells of a sterilised 96-well culture plate (Corning Inc., Corning, NY, USA) containing $200 \mu$ l of culture medium (CM) [Leibovitz's 15 (L-15) medium (Sigma-Aldrich) supplemented with $100 \mathrm{U} / \mathrm{ml}$ penicillin, $0.1 \mathrm{mg} / \mathrm{ml}$ streptomycin, $10 \mathrm{mM} \mathrm{HEPES}$ buffer (Sigma-Aldrich), and $10 \mathrm{mM} \mathrm{D-}$ glucose (Wako Pure Chemical Co., Osaka, Japan)]. The plates were then incubated at $25^{\circ} \mathrm{C}$.

The CM was changed every $24 \mathrm{~h}$. After brain slices were cultured for $72 \mathrm{~h}$, they were fixed with $4 \%$ paraformaldehyde solution in phosphate buffered saline (PBS) for $1 \mathrm{~h}$ for immunocytochemistry.

\section{Treatment of brain slices with11-KT or E2}

First, 10 mM 11-KT or E2 was prepared in $99.5 \%$ ethanol (Wako Pure Chemical Co.). In the experimental groups, after 11-KT and E2 were diluted with $\mathrm{CM}$, they were added to a 96-well plate at final concentrations of $1,10,100$, and $1000 \mathrm{nM}$ for $11-\mathrm{KT}$ and $10 \mathrm{nM}$ for E2. For the control groups, ethanol was added at the same concentration as that of the corresponding experimental group. The slices at the TN were cut into left and right halves (Fig. 1). One half of the brain slice was treated with either 11-KT or E2 (11-KT or E2 group) and the other half was treated with ethanol (control group). We used 3-5 animals for the respective concentrations of 11-KT and four animals for $10 \mathrm{nM}$ E2.

\section{Treatment of brain slices with IGF-1}

A solution of $50 \mu \mathrm{M}$ IGF-1 (IGF-1 Gilthead Seabream, PROSPEC, Hamada, Israel) was prepared using CM. Then, IGF-1 was added to a 96-well plate at a final concentration of 10 or $100 \mathrm{nM}$. For the control group, an equal volume of $\mathrm{CM}$ was added to each well. Slices at the TN were cut into left and right halves, which were used for the IGF-1 and control groups, respectively. Thus, IGF-1-treated and control slices originated from the same fish. We used slices from 3-5 animals for the respective concentrations of IGF-1.

\section{Treatment of brain slices with 11-KT and/or one of the inhibitors}


The following inhibitors were used in this study: IGF-1R inhibitor (BMS-754807; Funakoshi Co. Ltd., Tokyo, Japan), PI3K inhibitor (LY294002; Wako Pure Chemical Co.), AKT inhibitor (GDC-0068; Funakoshi Co. Ltd.), mTOR inhibitor (Rapamycin, Funakoshi Co. Ltd.), ERK inhibitor (U0126; Cosmo Bio Co. Ltd.), and JAK inhibitor (InSolution ${ }^{\text {TM }}$ JAK inhibitor I, Calbiochem, San Diego, CA, USA).

The right and left halves of brain slices were used. In 'the control group', the slice was incubated in the CM containing solvent (ethanol, DMSO, or saline) at the same concentration as that used to dissolve the respective inhibitor and 11-KT. In 'the inhibitor group', the slice was incubated in CM containing one of the inhibitors and the solvent to dissolve 11-KT. In 'the 11-KT group', the slice was incubated with $10 \mathrm{nM} 11-$ $\mathrm{KT}$, and the solvent was added at the same concentration as that used to dissolve the respective inhibitor. In 'the 11-KT + inhibitor group', the slices were incubated in CM containing $10 \mathrm{nM} 11-\mathrm{KT}$ and one of the inhibitors.

The slices for 'control' and 'inhibitor' groups originated from the same animal. Similarly, the slices for '11$\mathrm{KT}$ ' and '11-KT + inhibitor' groups originated from the same animal. We used 10-14 animals for the two pairs of the following four groups: the 'control', 'inhibitor', '11-KT', and '11-KT- and inhibitor' groups.

\section{Immunostaining with anti-GnRH3 antibodies.}

The brain slices at $72 \mathrm{~h}$ after cultivation were fixed with $4 \%$ paraformaldehyde. They were washed gently with PBS three times. Immunocytochemistry with an anti-GnRH3 antibody was performed using a custom-made rabbit anti-GnRH3 antibody ( $\times 7000$, produced by Protein Purify, Isesaki, Japan) and Alexa 555-labelled goat anti-rabbit IgG ( $\times 500$, Thermo Fisher Scientific, Ltd.). For nuclear staining, Hoechst 33342 solution (1000x, Wako Pure Chemical Co.) was used. After staining, coverslips were mounted with ProLong Gold antifade reagent (Thermo Fisher Scientific, Ltd.). The stained slices were observed using a confocal laser microscope (LSM 5 PASCAL, Carl-Zeiss AG, Jena, Germany). Since GnRH3 neurons are located close to each other and counting them accurately is difficult on a single sectional image, we obtained images in the z-stack with the confocal laser microscope and counted the actual number of cells in each slice. The total number of GnRH3 neurons per animal was calculated by summing the number of $\mathrm{GnRH} 3$ neurons on each half side of the brain slices (2-4 slices) originating from the same animal.

\section{Immunostaining with anti-IGF-1R and GnRH3 antibodies}

Sexually mature female tilapia of approximately equal size $(n=3$, approximately $30 \mathrm{~g}$ in body weight and $15 \mathrm{~cm}$ in total length) were used. 11-KT was dissolved in sesame oil at a concentration of $1.0 \mathrm{mg} / \mathrm{ml}$. Three mature females were intraperitoneally injected with $11-\mathrm{KT}$ at $5 \mu \mathrm{g} / \mathrm{g}$ body weight (11-KT-treated females). After the injections, the fish were maintained individually in 50-L glass tanks. Two days after the injection, they were deeply anaesthetized with $0.2 \% \mathrm{MS} 222$ and perfused transcardially with saline, followed by $4 \%$ paraformaldehyde in PBS ( $\mathrm{pH}$ 7.3). The brains were then removed and post-fixed in the same fixative overnight. Fixed brains were washed with PBS and immersed in 0.1 M PBS containing $20 \%$ 
sucrose overnight. Serial frontal sections (20- $\mu \mathrm{m}$ thickness) or mirror-image neighbouring frozen sections were cut on a cryostat (CM-3050-S, Leica Microsystems, Wetzlar, Germany), mounted onto MAS-coated glass slides (Matsunami Glass, Osaka, Japan), and stored at $20^{\circ} \mathrm{C}$.

The sections were stained either with one of the antibodies against GnRH3 $(\times 7000)$ and IGF-1R $(\times 1000$, rabbit polyclonal antibody, MyBioSource. Com., San Diego, CA, USA). Since these two antibodies were polyclonal antibodies raised in rabbits, a pair of mirror-image frozen sections was immunostained separately with those antibodies. The sections were incubated overnight with one of the antibodies. After washing with PBS, they were incubated with either Alexa Fluor 488- or 555-conjugated goat anti-rabbit IgG (500x, ThermoFisher Scientific, Ltd.). Hoechst 3334 solution (1000x) was used for nuclear staining. Finally, coverslips were mounted with ProLong Gold antifade reagent. Stained slices were observed using a confocal laser microscope.

\section{Real-time qRT-PCR analysis}

Nine animals were used in this experiment. Brain slices at the TN were cut into left and right halves, which were used for the 11-KT (10 nM) and control groups, respectively. Thus, 11-KT-treated and control slices originated from the same fish. The RNA samples were collected from the slices $1 \mathrm{~h}$ after the treatment. Reverse transcription and real-time qRT-PCR were performed as reported previously (Tsutiya et al., 2015, 2017). Cytoskeletal $\beta$-actin mRNA was used as a reference for qRT-PCR assays. The primer pairs used are as follows; CTGTGGAGAGCGAGGCTTTT and TTGGGAGTCTTGACAGGTGC for $l g f-1$, and AGCCAACAGGGAGAAGATG CCGGAGTCCATGACGATAC for $\beta$-actin.

\section{Statistical analysis}

Numerical data are presented as means \pm standard error of the mean. The significance of differences between the control and experimental groups (Fig. 2, 3, and 4) was determined by the Student's paired $t$ test. In experiments involving treatment with 11-KT and/or inhibitors (Fig. 5, 7, and 8), the significance of differences among groups was determined by a one-way ANOVA, followed by Tukey-Kramer post-hoc test at $p<0.05$. Additionally, when comparing the number of $\mathrm{GnRH} 3$ neurons in both halves of the brain slices originating from the same fish, a paired Student's $t$-test was used. Therefore, when comparing GnRH3 neuron numbers between the control and inhibitor groups, as well as the 11-KT and 11-KT and inhibitor groups, a paired Student's $t$-test was used. However, when comparing them in halves originating from different fish, an unpaired Student's $t$-test was used. In both cases, $p<0.05$ was considered statistically significant.

\section{Declarations}

\section{Data availability}

The data that support the findings of this study are available from the corresponding author upon reasonable request 


\section{Author contributions}

R.O-K. conceived and designed the experiments, and wrote the manuscript. A.O., S.I., R.K., Y.N. and S.S. carried out the experiments and data analysis. T.K. and Y-C.T contributed to sample preparation and aided in interpretation of results and writing of manuscript. All authors reviewed the manuscript.

\section{Acknowledgements}

We would like to acknowledge Dr. Satoshi Ogawa and Prof. Ishar S. Parhar's (Jeffrey Cheah School of Medicine and Health Sciences and the Brain Research Institute, Monash University, Malaysia) for kindly giving us their protocol for cultivating brain slices of tilapia. We also thank Prof. Hwang (Academia Sinica, Taiwan) for his helpful supports and useful advice.

\section{Competing interests}

The authors declare no competing interests.

\section{References}

1. Liu, H. et al. Sexual plasticity: A fishy tale. Mol. Reprod. Dev. 84(2), 171-194 (2017). 10.1002/mrd.22691, Pubmed:27543780

2. Godwin, J. Neuroendocrinology of sexual plasticity in teleost Fishes. Front. Neuroendocrinol. 31(2), 203-216 (2010). 10.1016/j.yfrne.2010.02.002, Pubmed:20176046

3. Senior, A. M., Nat Lim, J. \& Nakagawa, S. The fitness consequences of environmental sex reversal in fish: a quantitative review. Biol. Rev. Camb. Philos. Soc. 87(4), 900-911 (2012). 10.1111/j.1469185X.2012.00230.x, Pubmed:22540898

4. Bass, A. H. \& Grober, M. S. Social and neural modulation of sexual plasticity in teleost fish. Brain Behav. Evol. 57(5), 293-300 (2001). 10.1159/000047247, Pubmed:11641565

5. Munakata, A. \& Kobayashi, M. Endocrine control of sexual behavior in teleost fish. Gen. Comp. Endocrinol. 165(3), 456-468 (2010). 10.1016/j.ygcen.2009.04.011, Pubmed:19393660

6. Okubo, K., Miyazoe, D. \& Nishiike, Y. A conceptual framework for understanding sexual differentiation of the teleost brain. Gen. Comp. Endocrinol. 284, 113129 (2019). 10.1016/j.ygcen.2019.02.020

7. Clemens, H. P. \& Inslee, T. The production of unisexual broods by tilapia mossambica sex-reversed with methyltestosterone. Trans. Am. Fish. Soc. 97(1), 18-21 (1968). 10.1577/15488659(1968)97[18:TPOUBB]2.0.CO;2

8. Kobayashi, T., Kajiura-Kobayashi, H., Guan, G. \& Nagahama, Y. Sexual dimorphic expression of DMRT1 and Sox9a during gonadal differentiation and hormone-induced sex reversal in the teleost 
fish Nile tilapia (Oreochromis niloticus). Dev. Dyn. 237(1), 297-306 (2008). 10.1002/dvdy.21409, Pubmed:18095345

9. ljiri, S. et al. Sexual dimorphic expression of genes in gonads during early differentiation of a teleost fish, the Nile tilapia Oreochromis niloticus. Biol. Reprod. 78(2), 333-341 (2008).

10.1095/biolreprod.107.064246, Pubmed:17942796

10. Wang, D. S. et al. Foxl2 up-regulates aromatase gene transcription in a female-specific manner by binding to the promoter as well as interacting with ad4 binding protein/steroidogenic factor 1. Mol. Endocrinol. 21(3), 712-725 (2007). 10.1210/me.2006-0248, Pubmed:17192407

11. Wang, D. S. et al. Doublesex- and Mab-3-related transcription factor-1 repression of aromatase transcription, a possible mechanism favoring the male pathway in tilapia. Endocrinology 151(3), 1331-1340 (2010). 10.1210/en.2009-0999, Pubmed:20056824

12. Sun, L. N. et al. Transdifferentiation of differentiated ovary into functional testis by long-term treatment of aromatase inhibitor in Nile tilapia. Endocrinology 155(4), 1476-1488 (2014). 10.1210/en.2013-1959, Pubmed:24437491

13. Shi, $\mathrm{H}$. et al. Blockage of androgen and administration of estrogen induce transdifferentiation of testis into ovary. J. Endocrinol. 233(1), 65-80 (2017). 10.1530/JOE-16-0551, Pubmed:28148717

14. Kuramochi, A., Tsutiya, A., Kaneko, T. \& Ohtani-Kaneko, R. Sexual dimorphism of gonadotropinreleasing hormone type-III (GnRH3) neurons and hormonal sex reversal of male reproductive behavior in Mozambique tilapia. Zoolog. Sci. 28(10), 733-739 (2011). 10.2108/zsj.28.733, Pubmed:21967220

15. Gorbman, A. \& Sower, S. A. Evolution of the role of GnRH in animal (Metazoan) biology. Gen. Comp. Endocrinol. 134(3), 207-213 (2003). 10.1016/j.ygcen.2003.09.018, Pubmed:14636626

16. Morgan, K. \& Millar, R. P. Evolution of GnRH ligand precursors and $\mathrm{GnRH}$ receptors in protochordate and vertebrate species. Gen. Comp. Endocrinol. 139(3), 191-197 (2004).

10.1016/j.ygcen.2004.09.015, Pubmed:15560865

17. Tsai, P. S. Gonadotropin-releasing hormone in invertebrates: structure, function, and evolution. Gen. Comp. Endocrinol. 148(1), 48-53 (2006). 10.1016/j.ygcen.2005.09.016, Pubmed:16256989

18. White, S. A., Kasten, T. L., Bond, C. T., Adelman, J. P. \& Fernald, R. D. Three gonadotropin-releasing hormone genes in one organism suggest novel roles for an ancient peptide. Proc. Natl Acad. Sci. U.S. A. 92(18), 8363-8367 (1995). 10.1073/pnas.92.18.8363, Pubmed:7667296

19. Parhar, I. S., Pfaff, D. W. \& Schwanzel-Fukuda, M. Gonadotropin-releasing hormone gene expression in teleosts. Brain Res. Mol. Brain Res. 41(1-2), 216-227 (1996). 10.1016/0169-328x(96)00099-x, Pubmed:8883955

20. White, R. B. \& Fernald, R. D. Genomic structure and expression sites of three gonadotropin-releasing hormone genes in one species. Gen. Comp. Endocrinol. 112(1), 17-25 (1998).

10.1006/gcen.1998.7125, Pubmed:9748399

21. Yamamoto N. Oka Y. Kawashima S.Lesions of gonadotropin-releasing hormone-immunoreactive terminal nerve cells; effects on the reproductive behavior of male dwarf gouramis. 
Neuroendocrinology 65(6), 403-12 (1997).Pubmed: 9208402

22. Abe, H. \& Oka, Y. Mechanisms of neuromodulation by a nonhypophysiotropic GnRH system controlling motivation of reproductive behavior in the teleost brain. J. Reprod. Dev. 57(6), 665-674 (2011). 10.1262/jrd.11-055e, Pubmed:22277963

23. Ogawa, S. et al. Immunoneutralization of gonadotropin-releasing hormone type-III suppresses male reproductive behavior of cichlids. Neurosci. Lett. 403(3), 201-205 (2006).

10.1016/j.neulet.2006.02.041, Pubmed:16787709

24. Narita, Y. et al. Androgen induced cellular proliferation, neurogenesis, and generation of GnRH3 neurons in the brain of mature female Mozambique tilapia. Sci. Rep. 8(1), 16855 (2018). 10.1038/s41598-018-35303-9, Pubmed:30442908

25. Kitahashi, T., Sato, H., Sakuma, Y. \& Parhar, I. S. Cloning and functional analysis of promoters of three GnRH genes in a cichlid. Biochem. Biophys. Res. Commun. 336(2), 536-543 (2005). 10.1016/j.bbrc.2005.08.122, Pubmed:16139796

26. Dantzer, B. \& Swanson, E. M. Mediation of vertebrate life histories via insulin-like growth factor-1. Biol. Rev. Camb. Philos. Soc. 87(2), 414-429 (2012). 10.1111/j.1469-185X.2011.00204.x, Pubmed:21981025

27. Neirijnck, Y., Papaioannou \& Nef. The insulin/IGF system in mammalian sexual development and reproduction. Int. J. Mol. Sci. 20(18), 4440 (2019). 10.3390/ijms20184440

28. Zempo, B., Kanda, S., Okubo, K., Akazome, Y. \& Oka, Y. Anatomical distribution of sex steroid hormone receptors in the brain of female medaka. J. Comp. Neurol. 521(8), 1760-1780 (2013). 10.1002/cne.23255, Pubmed:23124931

29. Ogawa, S. \& Parhar, I. S. Single-cell gene profiling reveals social status-dependent modulation of nuclear hormone receptors in GnRH neurons in a male cichlid fish. Int. J. Mol. Sci. 21(8), 2724 (2020). 10.3390/ijms21082724, Pubmed:32326396

30. Dyer, A. H., Vahdatpour, C., Sanfeliu, A. \& Tropea, D. The role of insulin-like growth factor 1 (IGF-1) in brain development, maturation and neuroplasticity. Neuroscience 325, 89-99 (2016).

10.1016/j.neuroscience.2016.03.056, Pubmed:27038749

31. Daftary, S. S. \& Gore, A. C. IGF-1 in the brain as a regulator of reproductive neuroendocrine function. Exp. Biol. Med. (Maywood) 230(5), 292-306 (2005). 10.1177/153537020523000503, Pubmed:15855296

32. Wolfe, A., Divall, S. \& Wu, S. The regulation of reproductive neuroendocrine function by insulin and insulin-like growth factor-1 (IGF-1). Front. Neuroendocrinol. 35(4), 558-572 (2014). 10.1016/j.yfrne.2014.05.007, Pubmed:24929098

33. Daftary, S. S. \& Gore, A. C. The hypothalamic insulin-like growth factor-1 receptor and its relationship to gonadotropin-releasing hormones neurons during postnatal development. J. Neuroendocrinol. 16(2), 160-169 (2004). 10.1111/j.0953-8194.2004.01149.x, Pubmed:14764003

34. DiVall, S. A. et al. Divergent roles of growth factors in the $\mathrm{GnRH}$ regulation of puberty in mice. J. Clin. Invest. 120(8), 2900-2909 (2010). 10.1172/JCl41069, Pubmed:20628204 
35. Dees, W. L., Hiney, J. K. \& Srivastava, V. K. IGF-1 influences gonadotropin-releasing hormone regulation of puberty. Neuroendocrinology (2021). 10.1159/000514217, Pubmed:33406521

36. Reinecke, M., Schmid, A., Ermatinger, R. \& Loffing-Cueni, D. Insulin-like growth factor I in the teleost Oreochromis mossambicus, the tilapia: gene sequence, tissue expression, and cellular localization. Endocrinologist 138(9), 3613-3619 (1997). 10.1210/endo.138.9.5375. Pubmed:9275043'

37. Caelers, A., Berishvili, G., Meli, M. L., Eppler, E. \& Reinecke, M. Establishment of a real-time RT-PCR for the determination of absolute amounts of IGF-I and IGF-II gene expression in liver and extrahepatic sites of the tilapia. Gen. Comp. Endocrinol. 137(2), 196-204 (2004). 10.1016/j.ygcen.2004.03.006, Pubmed:15158131

38. Ma, W. et al. Sex differences in the expression of GH/IGF axis genes underlie sexual size dimorphism in the yellow catfish (Pelteobagrus fulvidraco). Sci. China Life Sci. 59(4), 431-433 (2016). 10.1007/s11427-015-4957-6, Pubmed:26660095

39. Shao, P. et al. The interference of DEHP in precocious puberty of females mediated by the hypothalamic IGF-1/PI3K/Akt/mTOR signalling pathway. Ecotoxicol. Environ. Saf. 181, 362-369 (2019). 10.1016/j.ecoenv.2019.06.017, Pubmed:31212184

40. Balint, F. et al. Insulin-like growth factor 1 (IGF-1) increases GABAergic neurotransmission to GnRH neurons via suppressing the retrograde tonic endocannabinoid signaling pathway in mice. Neuroendocrinology (2020). 10.1159/000514043 Online. Ahead of print. Pubmed:33361699

41. Onuma, T. A. et al. Regulation of temporal and spatial organization of newborn GnRH neurons by IGF signaling in zebrafish. J. Neurosci. 31(33), 11814-11824 (2011). 10.1523/JNEUROSCI.6804-10.2011, Pubmed:21849542

42. Seger, R. \& Krebs, E. G. The MAPK signaling cascade. FASEB J. 9(9), 726-735 (1995). 10.1096/fasebj.9.9.7601337, Pubmed:7601337

43. Coolican, S. A., Samuel, D. S., Ewton, D. Z., McWade, F. J. \& Florini, J. R. The mitogenic and myogenic actions of insulin-like growth factors utilize distinct signaling pathways. J. Biol. Chem. 272(10), 6653-6662 (1997). 10.1074/jbc.272.10.6653, Pubmed:9045696

44. Tang, L. et al. IGF-1R promotes the expression of cyclin D1 protein and accelerates the G1/S transition by activating Ras/Raf/MEK/ERK signaling pathway. Int. J. Clin. Exp. Pathol. 10(12), 11652-11658 (2017). Pubmed:31966523

45. Allen, M. P. et al. Growth arrest-specific gene 6 (Gas6)/adhesion related kinase (Ark) signaling promotes gonadotropin-releasing hormone neuronal survival via extracellular signal-regulated kinase (ERK) and Akt. Mol. Endocrinol. 13(2), 191-201 (1999). 10.1210/mend.13.2.0230, Pubmed:9973250

46. Cariboni, A. et al. VEGF signalling controls GnRH neuron survival via NRP1 independently of KDR and blood vessels. Development 138(17), 3723-3733 (2011). 10.1242/dev.063362, Pubmed:21828096

47. Novaira, H. J., Ng, Y., Wolfe, A. \& Radovick, S. Kisspeptin increases GnRH mRNA expression and secretion in GnRH secreting neuronal cell lines. Mol. Cell. Endocrinol. 311(1-2), 126-134 (2009). 10.1016/j.mce.2009.06.011, Pubmed:19576263 
48. Wu, S. et al. Jak2 is necessary for neuroendocrine control of female reproduction. J. Neurosci. 31(1), 184-192 (2011). 10.1523/JNEUROSCI.2974-10.2011, Pubmed:21209203

49. Wu, S. \& Wolfe, A. Signaling of cytokines is important in regulation of GnRH neurons. Mol. Neurobiol. 45(1), 119-125 (2012). 10.1007/s12035-011-8224-y, Pubmed:22161498

50. Magni, P. et al. Leukemia inhibitory factor induces the chemomigration of immortalized gonadotropin-releasing hormone neurons through the independent activation of the Janus kinase/signal transducer and activator of transcription 3, mitogen-activated protein kinase/extracellularly regulated kinase $1 / 2$, and phosphatidylinositol 3-kinase/Akt signaling pathways. Mol. Endocrinol. 21(5), 1163-1174 (2007). 10.1210/me.2006-0270, Pubmed:17299136

51. Dozio, E. et al. Expression of functional ciliary neurotrophic factor receptors in immortalized gonadotrophin-releasing hormone-secreting neurones. J. Neuroendocrinol. 17(5), 286-291 (2005). 10.1111/j.1365-2826.2005.01309.x, Pubmed:15869563

52. Tsutiya A. et al.Mouse pups lacking collapsin response mediator protein 4 manifest impaired olfactory function and hyperactivity in the olfactory bulb. Eur J Neurosci. 42(6), 2335-45 (2015). doi: 10.1111/ejn.12999, PMID: 26118640

53. Tsutiya A. et al. Human CRMP4 mutation and disrupted Crmp4 expression in mice are associated with ASD characteristics and sexual dimorphism. Sci. Rep. 7(1), 16812 (2017). doi: 10.1038/s41598017-16782-8, PMID: 29196732

\section{Figures}




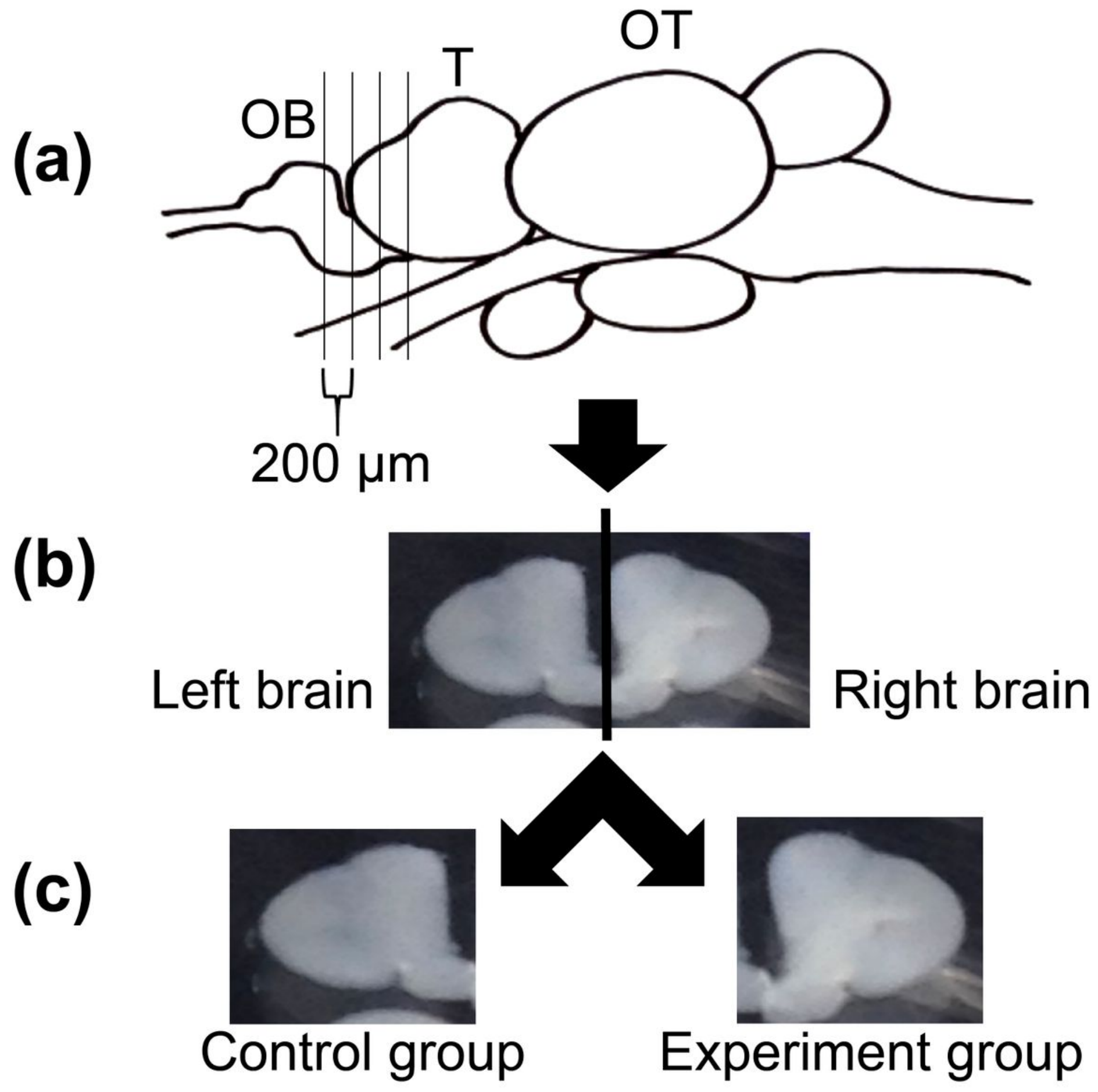

Figure 1

Brain slices of female tilapia. (a) A lateral view of the brain indicating the levels of the brain slices. (b) A cross-sectional slice of the brain cut at the terminal nerve. (c) Each brain slice was cut at the midline into right and left halves, which were used for the control and experimental groups, respectively. OB, olfactory bulb. T, telencephalon. OT, optic tectum. 
(a)

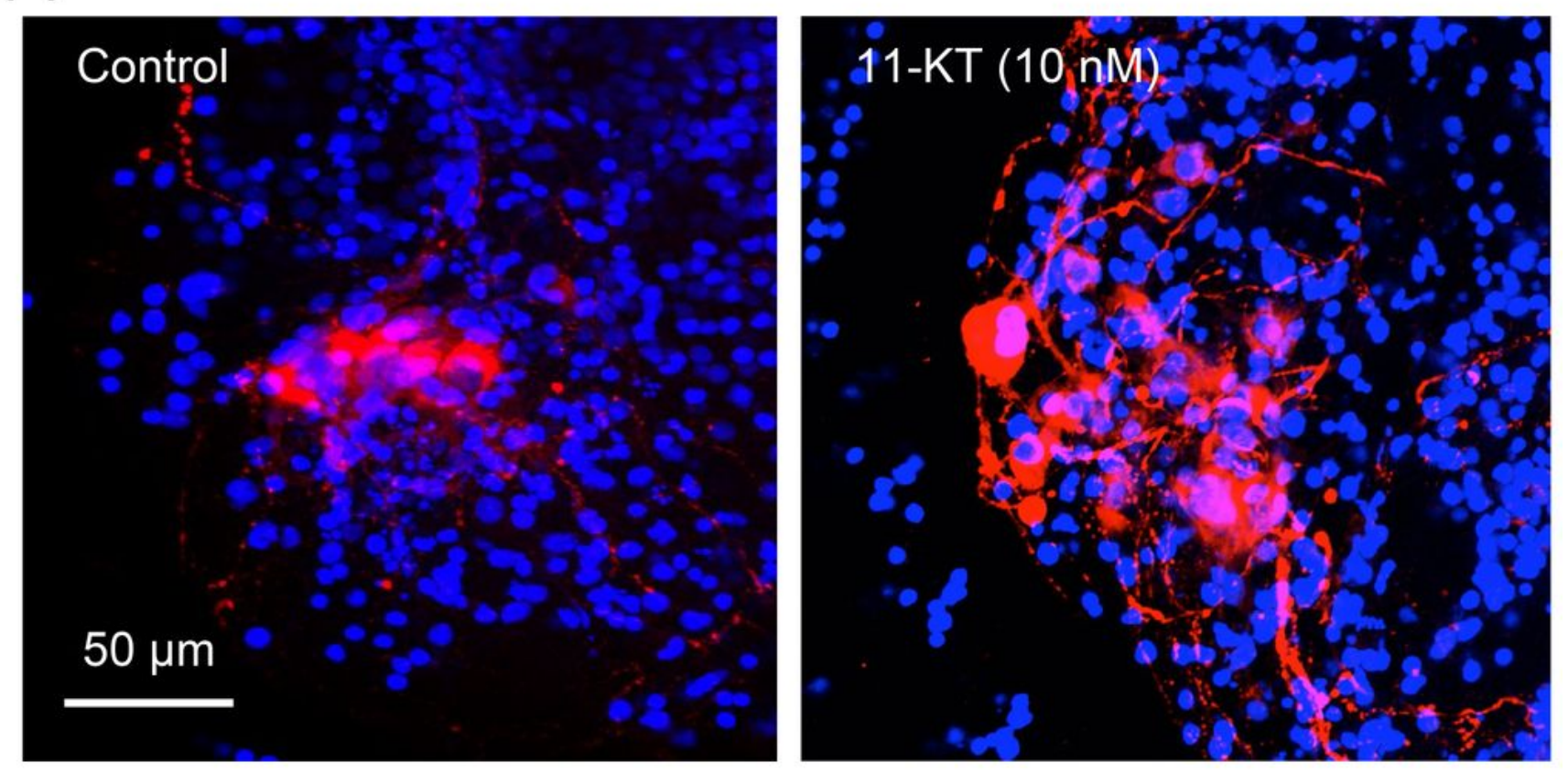

(b)

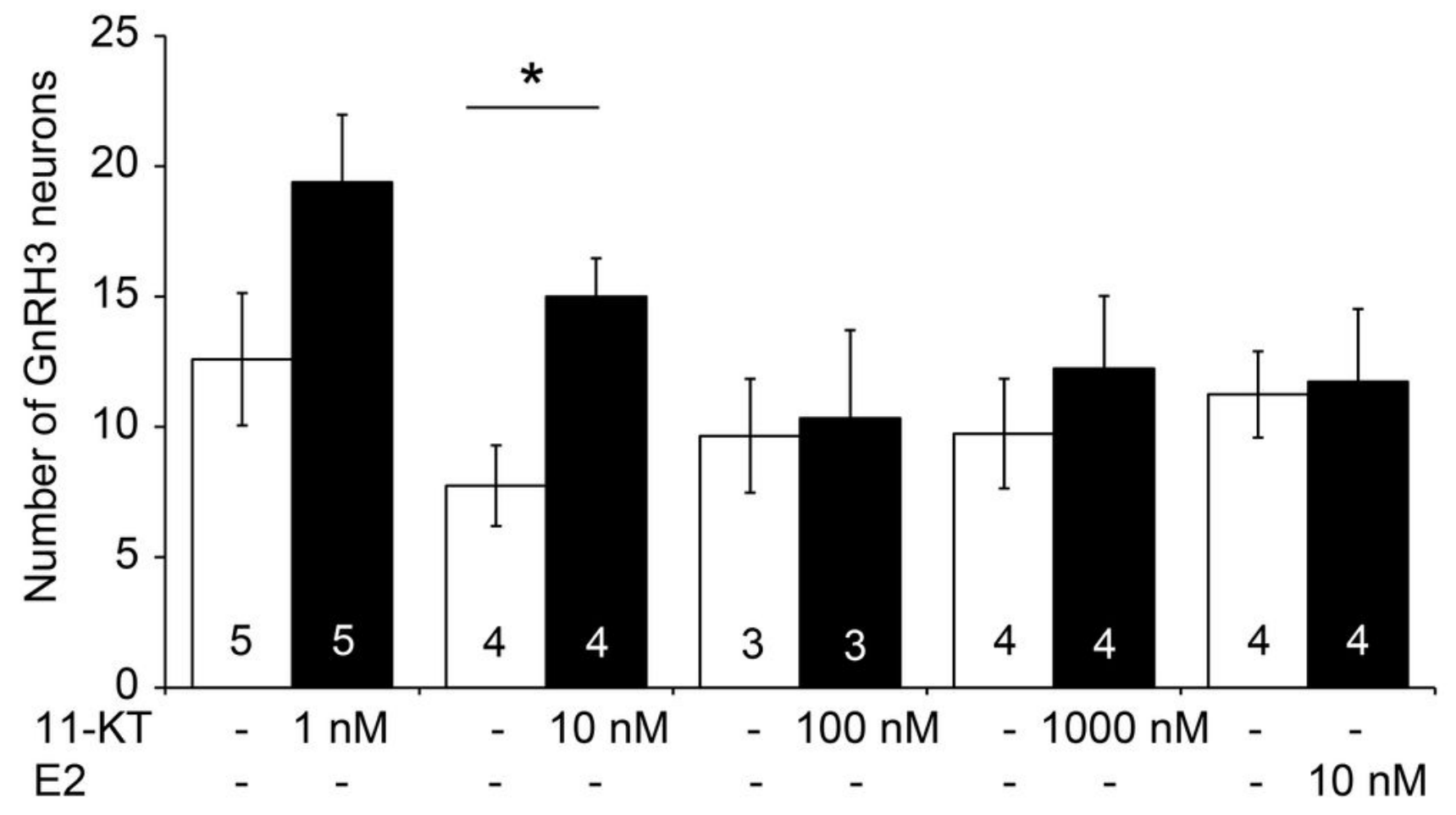

Figure 2

(a) Representative images of immunocytochemistry with gonadotropin-releasing hormone 3 (GnRH3) antibody. GnRH3 neurons (red) in control (left) and 11-ketotestosterone (11-KT)-treated (right) brain slices. Cell nuclei (blue) were stained with Hoechst 33342. (b) The numbers of GnRH neurons. White and black bars indicate the numbers of GnRH3 neurons in control slices and those treated with 11-KT $(1,10$, 100 and $1000 \mathrm{nM})$ and E2 (10 nM) (scale bar, $50 \mu \mathrm{m})$. An asterisk indicates a significant difference at 
$p<0.05$ (paired Student t-test). Numerals in bars indicate the numbers of animals used for preparing brain slices. Data are expressed as the mean \pm standard error of the mean.

(a)

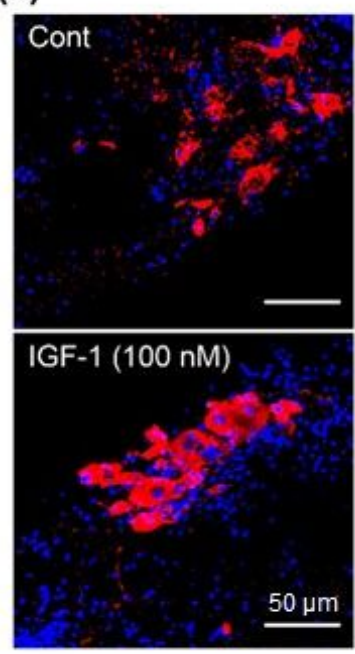

(b)

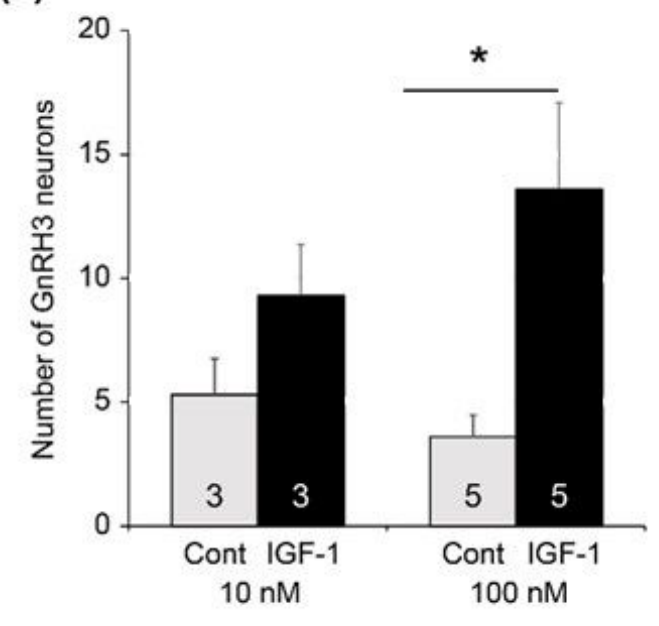

\section{Figure 3}

(a) Representative images of gonadotropin-releasing hormone 3 (GnRH3) neurons (red) in control (cont) and insulin-like growth factor 1 (IGF-1)-treated (100 nM) slices. Cell nuclei (blue) were stained with Hoechst 33342. (b) The numbers of GnRH3 neurons in control (Cont) and IGF-1 (10 nM and 100 nM)treated slices (scale bar, $50 \mu \mathrm{m}$ ). An asterisk indicates a significant difference at $\mathrm{p}<0.05$ (paired Student ttest). Numerals in bars indicate the numbers of animals used for preparing slices. Data are expressed as the mean \pm standard error of the mean. 


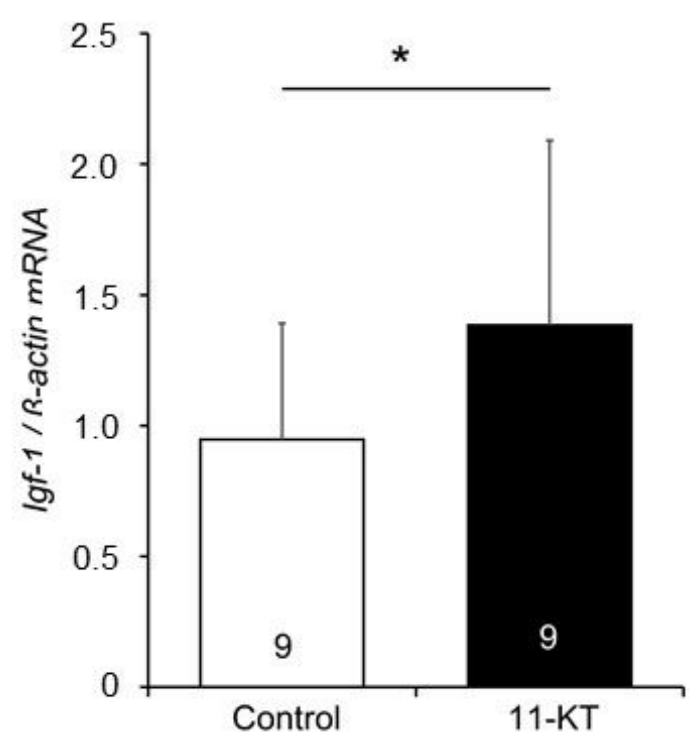

Figure 4

Changes in expressions of insulin-like growth factor 1 (Igf-1) mRNA in control and 11-ketotestosteronetreated slices $1 \mathrm{~h}$ after the treatment. An asterisk indicates a significant difference at $p<0.05$ (paired Student's t-test). Numerals in bars indicate the numbers of animals used for preparing slices. Data are expressed as the mean \pm standard error of the mean. 
(a)
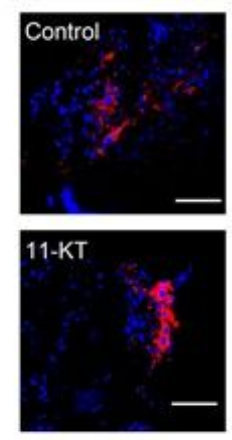

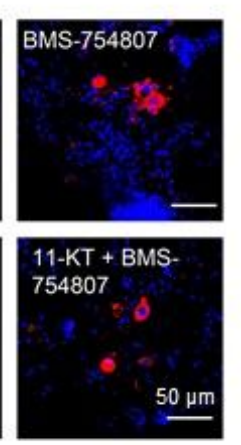

(b)

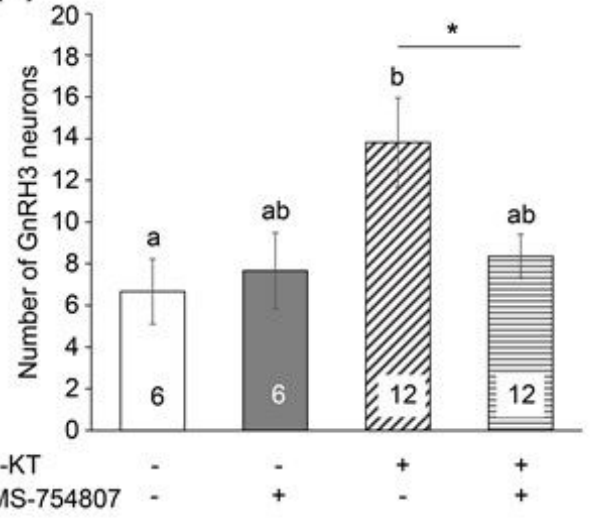

\section{Figure 5}

(a) Representative images of gonadotropin-releasing hormone 3 ( $\mathrm{GnRH} 3$ ) neurons (red) in the following four groups of brain slices: control, insulin-like growth factor 1 receptor (IGF-1R) inhibitor (20 $\mu \mathrm{M}$ BMS754807)-treated, 11-ketotestosterone (11-KT)-treated (10 nM), and 11-KT- (10 nM) and IGF-1R inhibitor (20 $\mu \mathrm{M}$ BMS-754807 )-treated groups. Control and inhibitor-treated slices originated from the same animals, while 11-KT-treated slices and 11-KT- and inhibitor-treated slices originated from the same animals. Cell nuclei (blue) were stained with Hoechst 33342. (b) The numbers of GnRH3 neurons counted in the four groups of brain slices. Different letters indicate significant differences as determined by a one-way analysis of variance followed by Tukey-Kramer post-hoc test at $p<0.05$. Numerals in bars indicate the numbers of animals used for preparing slices. An asterisk indicates a significant difference at $p<0.05$ (paired Student's t-test). Data are expressed as the mean \pm standard error of the mean. 


\section{(a) $\mathrm{GnRH} 3$ (red)}

(b) IGF-1recptor (green)
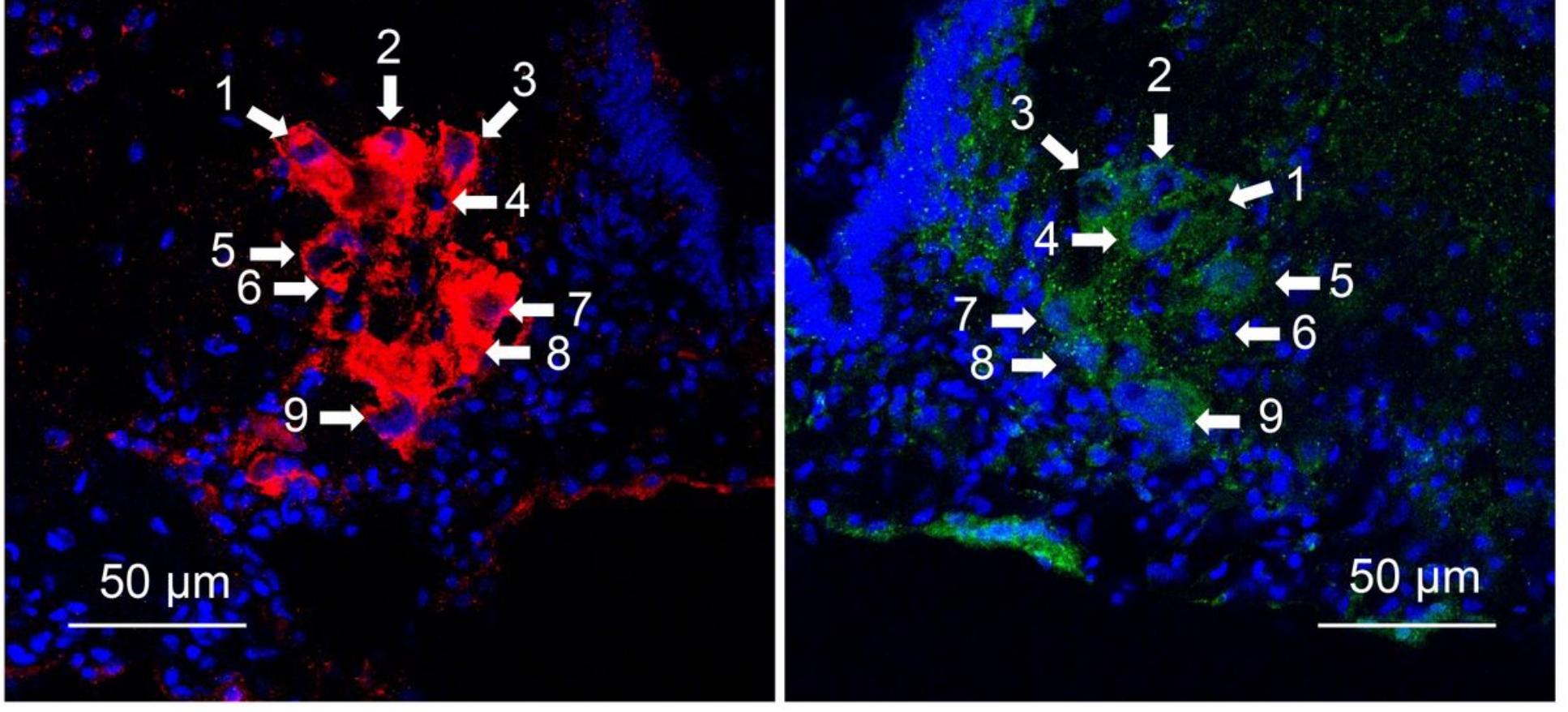

\section{Figure 6}

Representative images of gonadotropin-releasing hormone $3(\mathrm{GnRH})$ neurons [red in (a)] and insulin-like growth factor 1 receptor immunoreactivities [green in (b)] on a pair of mirror-image frozen sections from 11-ketotestosterone-injected females. The same numerals in respective pictures indicate the same cells appearing on the two sections. Cell nuclei (blue) were stained with Hoechst 33342. 
(a)
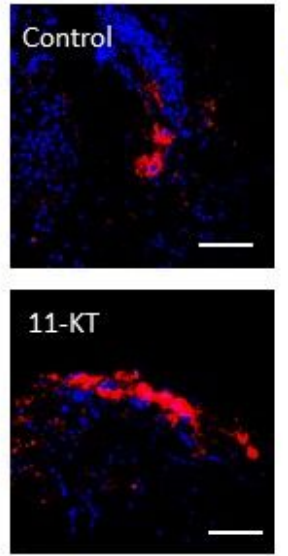

(b)
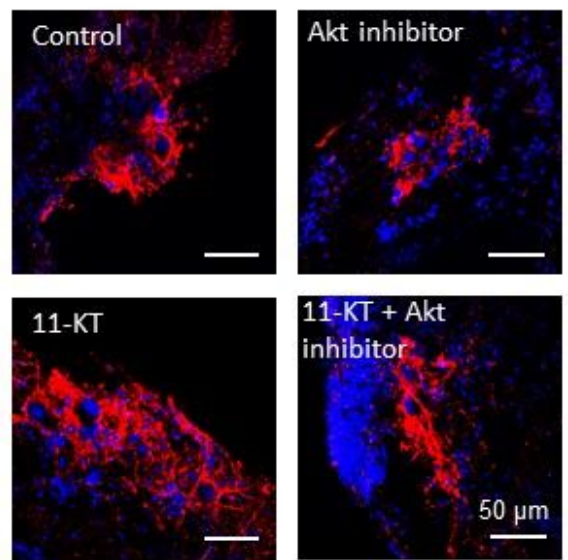

11-KT + PI3K

inhibitor
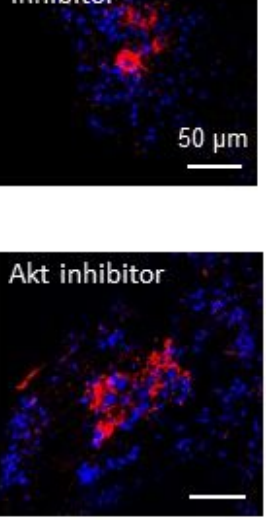

11-KT + Akt

inhibitor

$50 \mu \mathrm{m}$

(c)
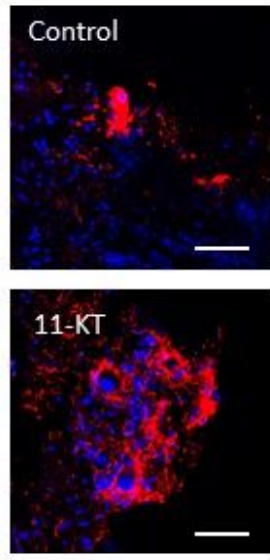

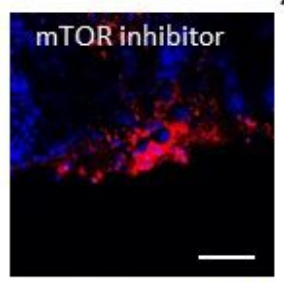

11-KT + mTOR

inhibitor

11-KT

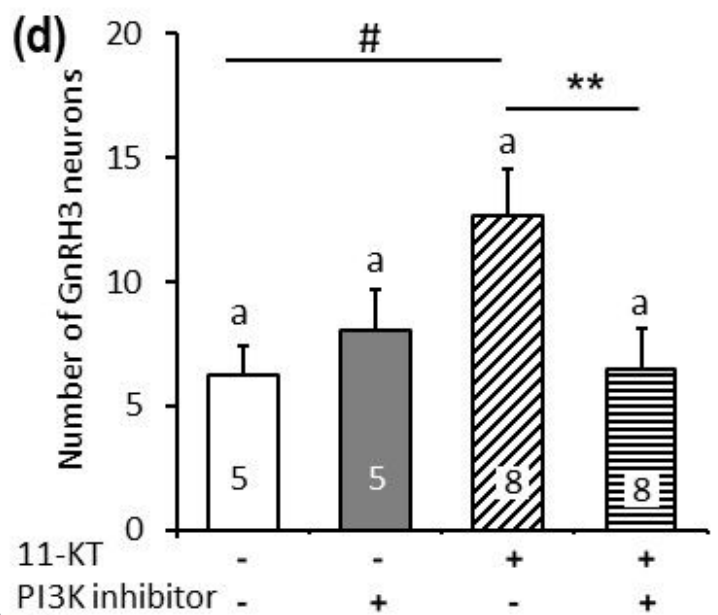

(e)

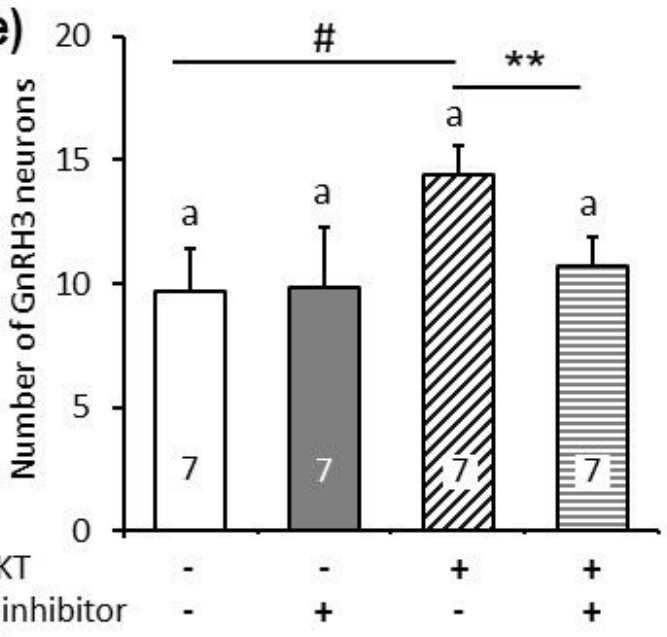

11-KT

(f)

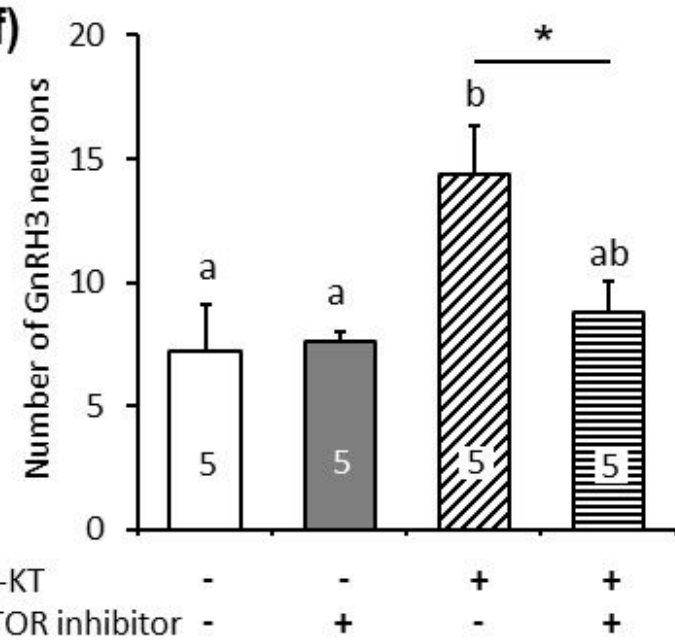

Figure 7

(a) (c) Representative images of gonadotropin-releasing hormone 3 ( $\mathrm{GnRH} 3$ ) neurons (red) in the following four groups of brain slices: control, inhibitor-treated, 11-ketotestosterone (11-KT)-treated (10 $\mathrm{nM})$, and 11-KT- (10 nM) and inhibitor-treated groups. Cell nuclei (blue) were stained with Hoechst 33342. (d) (f) The numbers of GnRH3 neurons in the four groups. Control and inhibitor-treated slices originated from the same animals, while 11-KT-treated slices and 11-KT and inhibitor-treated slices originated from 
the same animals. Inhibitors used in (a) (c) and (d) (f) were as follows: (a, d) phosphoinositide 3kinase (PI3K) inhibitor (LY294002), (b, e) AKT inhibitor (GDC-0068), (c, f) mechanistic target of rapamycin (mTOR) inhibitor (rapamycin). Different letters indicate significant differences as determined by a oneway analysis of variance followed by Tukey-Kramer post-hoc test at $p<0.05$. Numerals in bars indicate the numbers of animals used for preparing slices. * and ** indicate significant differences at $p<0.05$ and $p<0.01$, respectively (paired Student's t-test). \# indicates a significant difference at $p<0.05$ (unpaired Student t-test). Data are expressed as the mean \pm standard error of the mean.

(a)
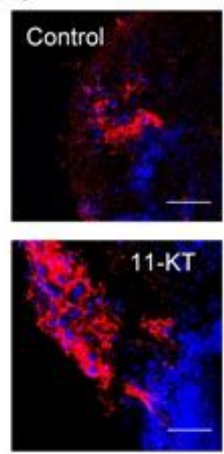

(b)

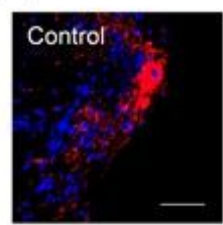

11-KT

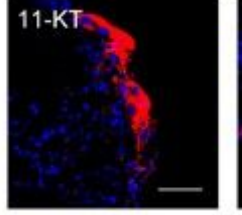

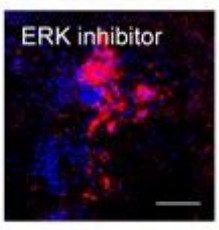

11-KT + ERK inhibitor

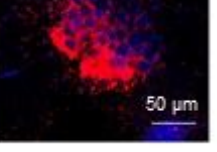

$50 \mu \mathrm{m}$

(c)

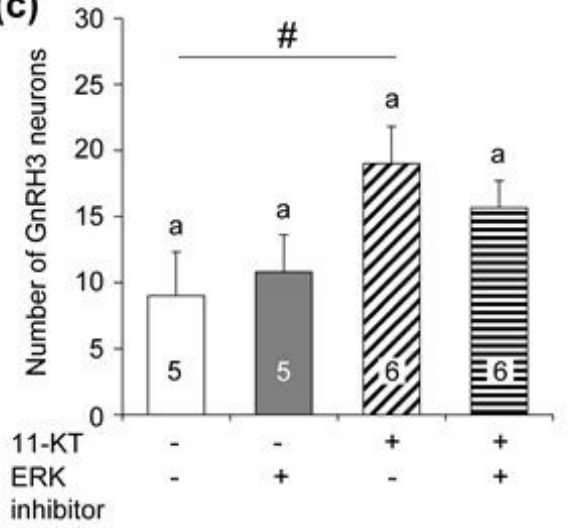

(d)

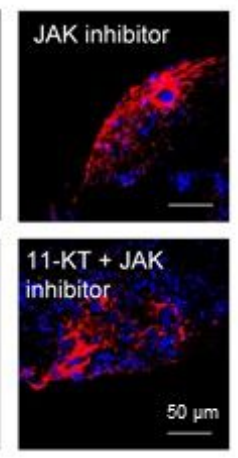

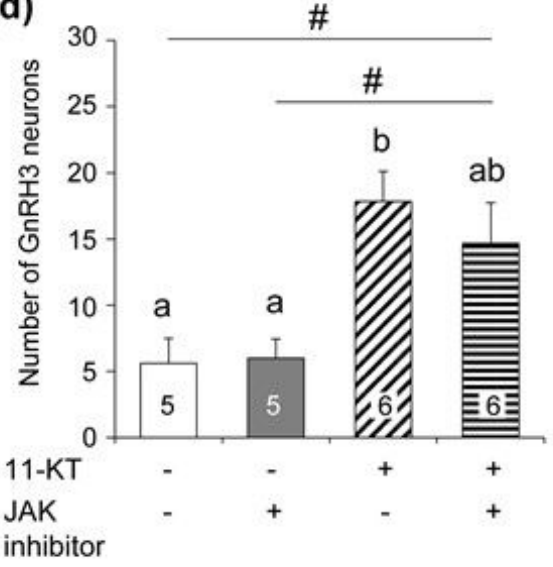

\section{Figure 8}

$(a, b)$ Representative images of gonadotropin-releasing hormone $3(\mathrm{GnRH} 3)$ neurons (red) in the following four groups of brain slices: control, inhibitor-treated, 11-ketotestosterone (11-KT)-treated (10 nM), and 11KT- (10 nM) and inhibitor-treated groups. Cell nuclei (blue) were stained with Hoechst 33342. (c, d) The numbers of $\mathrm{GnRH} 3$ neurons in the four groups. Control and inhibitor-treated slices originated from the same animals, while 11-KT-treated slices and 11-KT- and inhibitor-treated slices originated from the same animals. Inhibitors used in ( $a, c)$ and (b, d) were as follows: (a, c) ERK inhibitor (20 $\mu M$ U0126), (b, d) JAK inhibitor (100 nM, InSolutionTM JAK inhibitor 1). Different letters indicate significant differences as 
determined by a one-way analysis of variance followed by Tukey-Kramer post-hoc test at $p<0.05$. Numerals in bars indicate the numbers of animals used for preparing slices. \# indicates significant differences at $p<0.05$ in unpaired Student's t-test. Data are expressed as the mean \pm standard error of the mean. 